\title{
Maximum alternation rate in bi-stable perception occurs at equidominance: experiments and modeling
}

\author{
Asya Shpiro*1, Rubén Moreno-Bote ${ }^{1}$, Susan Bloomberg1,2, John Rinzel ${ }^{1,2}$ and \\ Nava Rubin ${ }^{1}$
}

Address: ${ }^{1}$ Center for Neural Science, New York University, New York, USA and ${ }^{2}$ Courant Institute of Mathematical Sciences, New York University, New York, USA

Email: Asya Shpiro* - avs203@nyu.edu

* Corresponding author

from Sixteenth Annual Computational Neuroscience Meeting: CNS*2007

Toronto, Canada. $7-12$ July 2007

Published: 6 July 2007

BMC Neuroscience 2007, 8(Suppl 2):P78 doi:10.1186/147I-2202-8-S2-P78

(c) 2007 Shpiro et al; licensee BioMed Central Ltd.

We present ambiguous stimuli, such as a pair of superimposed gratings exhibiting bi-stable depth ordering and binocular rivalry stimulus, to human subjects and measure the amount of time each of the two possible percepts dominates the other. Parameters of the stimuli are manipulated in order to strengthen gradually one of the percepts compared to the other, that is, to increase the fraction of time that this percept is dominant. For some choice of the parameters, the two percepts become equidominant: they dominate for the same fraction of time, or, equivalently, their mean dominance durations are the same. When the parameter which controls the strength of the percepts is varied from the equidominance value, its effect on the mean dominance duration for each percept is not the same. For the percept which becomes stronger, the mean dominance duration increases greatly in comparison to its value at equidominance, while for the percept which becomes weaker, the mean dominance duration is only slightly reduced. This result implies that as a function of the parameter that controls percept strength, the alternation rate between the two interpretations reaches a maximum at the equidominance point. We show that these features naturally arise in a bi-stable energy-based attractor model [1] where parameter manipulations produce symmetrical deformations of the energy landscape. Based on this formalism, we construct a two-population ratebased model with divisively normalized inputs, which produces behavior qualitatively similar to the one observed experimentally. A general class of neuronal competition models which describe rivalry during ambiguous stimulus presentation $[2,3]$ exhibits the described behavior as well, as long as divisively normalized inputs are introduced. We calculate the entropy of a binary system in which probabilities are equal to the fraction of dominance of each state and show that it correlates with the alternation rate between the states in both experiments and models, suggesting that the alternation rate is a reflection of the uncertainty present in the system due to ambiguous stimulation.

\section{References}

I. Moreno-Bote R, Rinzel J, Rubin N: Noise-induced alternations in an attractor network model of perceptual bi-stability. J Neurophys 2007 in press.

2. Laing CR, Chow CC: A spiking neuron model for binocular rivalry. J Comput Neurosci 2002, I 2:39-53.

3. Shpiro A, Curtu R, Rinzel J, Rubin N: Dynamical characteristics common to neuronal competition models. J Neurophysiol 2007, 97:462-473. 